Article

\title{
Immune-Enhancing Effects of Red Platycodon grandiflorus Root Extract via p38 MAPK-Mediated NF-KB Activation
}

\author{
Eun-Jung Park ${ }^{1,2} \mathbb{1}$, You-Suk Lee ${ }^{1,2}$, Sung Min Kim ${ }^{1}$, Ah Jin Jung ${ }^{2}$, Jeong-Hyun Yoo ${ }^{2} \mathbb{D}$, \\ Sung-Hyen Lee ${ }^{3}$, hyun Cheol Jeong ${ }^{4}$ and hae-Jeung Lee ${ }^{1,2, *}$ \\ 1 Department of Food and Nutrition, College of BioNano Technology, Gachon University, Gyeonggi-do 13120, \\ Korea; ejpark@gachon.ac.kr (E.-J.P.); ysleeyun@gachon.ac.kr (Y.-S.L.); kimsm127@gc.gachon.ac.kr (S.M.K.) \\ 2 Institute for Aging and Clinical Nutrition Research, Gachon University, Gyeonggi-do 13120, Korea; \\ dkwls2784@naver.com (A.J.J.); yjh8252@naver.com (J.-H.Y.) \\ 3 National Institute of Agricultural Sciences, Rural Department Administration, Wanju, Jeonbuk 55365, Korea; \\ lshin@korea.kr \\ 4 Food R\&D Center, SK Bioland Co., Ltd., Ansan, Gyeonggi-do 13120, Korea; hyuncheolj@sk.com \\ * Correspondence: skysea@gachon.ac.kr or skysea1010@gmail.com; Tel.: +82-31-750-5968
}

Received: 21 July 2020; Accepted: 5 August 2020; Published: 7 August 2020

\begin{abstract}
Platycodongrandiflorus (PG) root extract has been widely used as an oriental herbal medicine. Red PG root extract (RPGE), which is made by steaming and drying PG several times, contains more saponin than raw (white) PG. Although RPGE has been known to have anti-inflammatory activity, the effects of RPGE on the immune-enhancing response remain unclear. In this study, we aimed to investigate the immune-enhancing effects of RPGE and its mechanism in macrophage cells and splenocytes. Our results revealed that cell proliferation of both macrophages and splenocytes correlate positively with the concentration of RPGE. Moreover, RPGE treatment increased the phagocytic activity of macrophage cells, as well as nitric oxide and cytokines production. Furthermore, RPGE induced phosphorylation of the p38 mitogen-activated protein kinase, which contributed to nuclear factor-kappa B activation. Thus, our findings suggest that RPGE may be a potential functional food for improving immune function.
\end{abstract}

Keywords: red platycodon grandiflorus root extract; immune-enhancing effect; p38 MAPK; NF-кB

\section{Introduction}

Herbal immunostimulants are generally categorized as natural compounds that can enhance immune responses by activating immune cells, such as macrophages [1,2]. By promoting the phagocytosis of macrophages, immunostimulants induce the secretion of cytokines, such as tumor necrosis factor-alpha (TNF- $\alpha$ ), interleukin-1 beta (IL-1 $\beta$ ), and interleukin-6 (IL-6), thereby improving immune function [3].

Nuclear factor-kappa B (NF-kB)-mediated cellular changes are known to be closely associated with both the innate and adaptive immune responses [4,5]. In innate immune cells, such as macrophages, NF- $\kappa B$ activation is initiated by the degradation of inhibitory kappa B alpha (I $\kappa$ B) proteins, following which the free NF- $\kappa B$ enters the nucleus to perform various downstream functions, including immune regulation and proliferation [4,6]. Generally, immunostimulants activate the immune cell function through specific binding with receptors [7]. Moreover, immunostimulants trigger several downstream signals, such as the NF-кB pathway, p38 mitogen-activated protein kinase (MAPK), and the c-Jun N-terminal kinase (JNK) pathway, increasing the secretion of nitric oxide (NO), as well as TNF- $\alpha$, IL-1 $\beta$, and IL-6 [1,7]. 
The spleen plays a role in the immune system by protecting against blood-derived antigens and removing aged red blood cells and damaged cells [8]. The populations of cells in the spleen are mainly composed of various immune lymphocytes, including T cell, B cell, and macrophages [9], which are widely used for immunomodulatory research.

Platycodon grandiflorus (PG) has a long history of being widely used as a traditional medicine [10]. It has been reported, inter alia, to reduce inflammation and improve liver function $[8,10]$. Saponins, which are part of single compounds of PG, have also been studied actively, mainly focusing on their anti-cancer properties $[10,11]$. Among them, platycodin D showed immunoadjuvant activity in ovalbumin-immunized mice [12] and increased serum cytokine levels in h22 hepatocellular carcinoma-bearing mice [11], providing the evidence of immune enhancing effect. Red PG, which is made by steaming and drying PG several times, increases the saponin content during processing when compared to raw (white) PG [8]. Red PG with a high content of platycodin D might have potential as a herbal immunostimulant, however no study has been conducted on the pharmacologic effects and molecular mechanisms of red PG in the immune-enhancing response. In this study, we aimed to evaluate the immune-enhancing effects of red PG extract (RPGE) in RAW 264.7 macrophage cells and mouse splenocytes. We also investigated its effects on the NF-KB and MAPK signaling pathways to determine the underlying mechanisms of the immune-enhancing effects of RPGE in immune cells. Thus, the results of our study provide novel insights regarding the immunomodulatory effects and the mechanism action of RPGE.

\section{Materials and Methods}

\subsection{Red Platycodon grandiflorus Root Extract (RPGE) Preparation}

RPGEs used in the experiments were supplied by SK Bioland (Ansan, Korea) in two types of formulation, concentrate and powder, which are representative formulations of functional foods. Both types of RPGEs were prepared by standard production processes. Briefly, Korean PG roots were washed twice and steamed for $2 \mathrm{~h}$. Following that, the roots were subjected to 4 cycles of drying for $24 \mathrm{~h}$ and steaming for $90 \mathrm{~min}$ and then dried for $72 \mathrm{~h}$ to prepare red PG. The red PG was mixed with $50 \%$ ethanol (Korea ethanol supplies company, Seoul, Korea) at a ratio of 1:15 $(w / v)$ and extracted at $80^{\circ} \mathrm{C}$ for $8 \mathrm{~h}$. The primary extract was recovered, and the remaining residues were subjected to a secondary extraction at $80^{\circ} \mathrm{C}$ for $8 \mathrm{~h}$ with $50 \%$ ethanol (ratio 1:15, w/v). All extracts were then mixed and filtered using a filter press. The filtered extract was concentrated at reduced pressure until the solid content reached $60 \%$ or more and subsequently sterilized to obtain RPGE-concentrate (RPGE-C). To prepare RPGE-powder (RPGE-P), RPGE-C was mixed with dextrin at a 1:1 ratio and then spray-dried.

\subsection{Cell Culture and Reagents}

RAW 264.7 cells purchased from the American Type Culture Collection (Manassas, VA, USA) and maintained in Dulbecco's Modified Eagle's Medium supplemented with $10 \%$ fetal bovine serum (FBS) and $1 \%$ antibiotic-antimycotic in $5 \% \mathrm{CO}_{2}$ at $37^{\circ} \mathrm{C}$. All cell culture reagents were obtained from GIBCO (Gaithersburg, MD, USA). SB203580 and SP600125 were purchased from Calbiochem (San Diego, CA, USA). Concanavalin A (Con A) and lipopolysaccharide (LPS) were obtained from Sigma-Aldrich (St. Louis, MO, USA).

\subsection{Splenocyte Isolation}

The five-week-old imprinting control region (ICR) male mice were purchased from Orient Bio Co. (Seongnam, Korea). The spleens were isolated and rinsed with RPMI 1640 medium. After that, the spleens were physically crushed and filtered through a $200 \mu \mathrm{m}$ cell strainer (BD Biosciences, San Jose, CA, USA) and centrifuged at $3000 \mathrm{rpm}$ for $10 \mathrm{~min}$ at $4{ }^{\circ} \mathrm{C}$. The cell pellets were resuspended and incubated for $5 \mathrm{~min}$ with lysis buffer to remove erythrocytes. The cells were then washed with RPMI 1640 medium and centrifuged (3000 rpm, $10 \mathrm{~min}, 4^{\circ} \mathrm{C}$ ) to obtain splenocytes. Isolated 
splenocytes were cultured for each experiment in RPMI 1640 medium supplemented with $10 \%$ FBS and $1 \%$ antibiotic-antimycotic in an atmosphere containing $5 \% \mathrm{CO}_{2}$ at $37^{\circ} \mathrm{C}$. The animal protocols used in this work were evaluated and approved by the Institutional Animal Care and Use Committee (IACUC) of the Gachon University (reference number: GIACUC-R2019019). They are in accordance with Korean Animal Protection Act (Act No. 16075).

\subsection{Cell Viability Assay}

RAW 264.7 cells or splenocytes isolated from mice were seeded at a concentration of $2 \times 10^{4}$ cells/well and $5 \times 10^{5}$ cells/well into 96-well plates, respectively. After $24 \mathrm{~h}$ (RAW 264.7 cells) or immediately (splenocytes), the cells were treated with various concentrations of RPGE-C or RPGE-P. For the splenocytes proliferation assay, Con A and LPS were treated as positive mitogens. After incubating for $24 \mathrm{~h}$, the Cell Counting Kit-8 (CCK-8, Dojindo Molecular Technologies, Inc., Kumamoto, Japan) solution was added $(10 \mu \mathrm{L} /$ well $)$ and cells were further incubated at $37^{\circ} \mathrm{C}$ for $2 \mathrm{~h}$. The absorbance of each well was measured at $450 \mathrm{~nm}$ on an Epoch Microplate Spectrophotometer (Biotek Inc., Winooski, VT, USA).

\subsection{Phagocytosis Assay}

The phagocytic ability was determined using the CytoSelect ${ }^{\mathrm{TM}}$ 96-well phagocytosis assay kit (Cell Biolabs, Inc., San Diego, CA, USA), according to the manufacturer's instructions. Briefly, RAW 264.7 cells were seeded at a concentration of $2 \times 10^{4}$ cells/well into 96-well plates. The cells were preincubated with the phagocytosis inhibitor (PI, $2 \mu \mathrm{M}$ of Cytochalasin D) or various concentrations of RPGE-C or RPGE-P for $24 \mathrm{~h}$ before addition of the E. coli suspension. After incubating for $4 \mathrm{~h}$, we performed a removal and blockage of external particles step. The internalized particles were detected at $450 \mathrm{~nm}$ using an Epoch Microplate Spectrophotometer (Biotek Inc., Winooski, VT, USA).

\subsection{Measurement of Nitric Oxide (NO) Production}

RAW 264.7 cells were seeded in 6-well plates at $4.0 \times 10^{5}$ cells/well. The cells were treated with various concentrations of RPGE-C or RPEG-P for $24 \mathrm{~h}$. NO levels in the media were assayed using the Griess Reagent System (Promega, Madison, WI, USA). Cell culture supernatant aliquots with a volume of $50 \mu \mathrm{L}$ were incubated with the substrate solution for $10 \mathrm{~min}$, followed by incubation avoiding light with the coloring solution for another $10 \mathrm{~min}$. The absorbance was measured at $520 \mathrm{~nm}$ using an Epoch Microplate Spectrophotometer (Biotek Inc., Winooski, VT, USA). NO production was determined by comparison to the dilution of a sodium nitrite standard.

\subsection{RNA Isolation and Real-Time Reverse Transcription-Polymerase Chain Reaction (RT-PCR)}

Total RNA was isolated using the easy-spin ${ }^{\mathrm{TM}}$ total RNA extraction kit (iNtRON Biotechnology, Gyeonggi-do, Korea), and cDNA was synthesized using the GoScript ${ }^{\mathrm{TM}}$ Reverse Transcriptase (Promega, Madison, WI, USA). Real-time PCR was performed on the synthesized cDNA using the TB Green ${ }^{\mathrm{TM}}$ Premix Ex Taq ${ }^{\mathrm{TM}}$ II (TaKaRa Bio, Otsu, Japan) on the ABI QuantStudio 3 (Applied Biosystems, Foster City, CA, USA). The primer sequences $\left(5^{\prime}-3^{\prime}\right)$ used in the experiments were shown in Table 1 . All gene expression values were normalized to $A c t b$ ( $\beta$-actin).

Table 1. The primer sequences $\left(5^{\prime}-3^{\prime}\right)$ used in the experiments.

\begin{tabular}{cccc}
\hline Genes & Forward Sequence & Reverse Sequence & Ref. \\
\hline Nos2 & GCGAAAGGTCATGGCTTCAC & CTGGTCCATGCAGACAACCT & This study \\
Ptgs 2 & CATCCCCTTCCTGCGAAGTT & GGCCCTGGTGTAGTAGGAGA & This study \\
Tnf- $\alpha$ & TGTCCCTTTCACTCACTGGC & CATCTTTTGGGGGAGTGCCT & {$[13]$} \\
$I L-1 b$ & AACTGTTCCTGAACTCAACTGT & GAGATTTGAAGCTGGATGCTCT & {$[14]$} \\
$I L-6$ & GGGACTGATGCTGGTGACAA & TCCACGATTTCCCAGAGAACA & {$[13]$} \\
$A c t b$ & GACGTTGACATCCGTAAAG & CAGTAACAGTCCGCCT & {$[2]$} \\
\hline
\end{tabular}




\subsection{Quantification of Cytokine Levels}

RAW 264.7 cells or mouse splenocytes were seeded in 6-well plates at $4.0 \times 10^{5}$ cells/well. The cells were treated with various concentrations of RPGE-C or RPEG-P for $24 \mathrm{~h}$. To determine the cytokine levels, media was collected, and IL-6 and IL-10 levels were measured using enzyme-linked immunosorbent assays kits (ELISA; R\&D Systems Inc., Minneapolis, MN, USA), according to the manufacturer's instructions.

\subsection{Luciferase Assay}

RAW 264.7 cells were cotransfected with the $3 \times \kappa \mathrm{B}-\mathrm{Luc}$ [15] and pNL1.1.TK vector using FuGENE ${ }^{\circledR}$ hD (Promega, Madison, WI, USA) for $24 \mathrm{~h}$. The transfected cells were then incubated with RPGEs $(0,20,100$, or $500 \mu \mathrm{g} / \mathrm{mL})$. After incubation for $24 \mathrm{~h}$, the cells were assayed using a Nano-Glo ${ }^{\circledR}$ Dual-Luciferase ${ }^{\circledR}$ Reporter Assay System (Promega, Madison, WI, USA) according to the manufacturer's instructions. The luciferase activity was detected using a GloMax ${ }^{\circledR}$ Discover Multimode Microplate Reader (Promega, Madison, WI, USA).

\subsection{Western Blot Analysis}

The crude extracts from RAW 264.7 cells were prepared using the lysis solution (PRO-PREPTM Protein Extraction Solution plus the halt ${ }^{\mathrm{TM}}$ phosphatase inhibitor cocktail, iNtRON Biotechnology and Thermo Scientific, Waltham, MA, USA, respectively). The crude extracts were separated on a $10 \%$ sodium dodecyl sulfate-polyacrylamide gel electrophoresis and probed with the indicated antibodies. Antibodies against p-NF-kB p65 (Ser536), NF-кB p65, phospho-IкB $\alpha$ (Ser32), phospho-p38 (Thr180/tyr182), p38, phospho-JNK (Thr183/Try185), and JNK were purchased from Cell Signaling Technology (Danvers, MA, USA). The $\beta$-actin antibody was purchased from Abcam (Cambridge, MA, USA). Secondary antibodies were obtained from Promega. ImageQuant LAS 500 (GE healthcare Life Sciences, Little Chalfont, UK) were used for visualization.

\subsection{Statistical Analysis}

All data are expressed as mean \pm SEM. Multiple comparisons of means among experimental groups were carried out with one-way ANOVA, followed by a post-hoc test using the GraphPad Prism 5 software (Graph Pad Software Inc., San Diego, CA USA). A $p$-value of $<0.05$ was considered statistically significant.

\section{Results}

\subsection{RPGE Increases Phagocytic Activity in RAW 264.7 Cells}

To assess whether the RPGE affects cell viability, we performed the CCK-8 assay. Both RPGE-C and RPGE-P were shown to have any cytotoxic effect on RAW 264.7 cells. Interestingly, the viable cell population was increased by RPGEs in a concentration-dependent manner (Figure 1a,b). To ascertain whether RPGE-induced cell proliferation in RAW 264.7 cells is related to macrophage activation, we first analyzed the phagocytic ability of RPGE treated cells. The phagocytic activity was monitored by measuring the amount of internalized E. coli in macrophages. RAW 264.7 cells were pretreated with PI or RPGEs $(20,100$, or $500 \mu \mathrm{g} / \mathrm{mL})$ for $24 \mathrm{~h}$ before addition of the E. coli suspension. After treatment with RPGE-C or RPGE-P at concentrations above $100 \mu \mathrm{g} / \mathrm{mL}$, the phagocytic activity of cells was found to be significantly increased than in cells treated with the vehicle control (VC) (Figure 2a,b). 


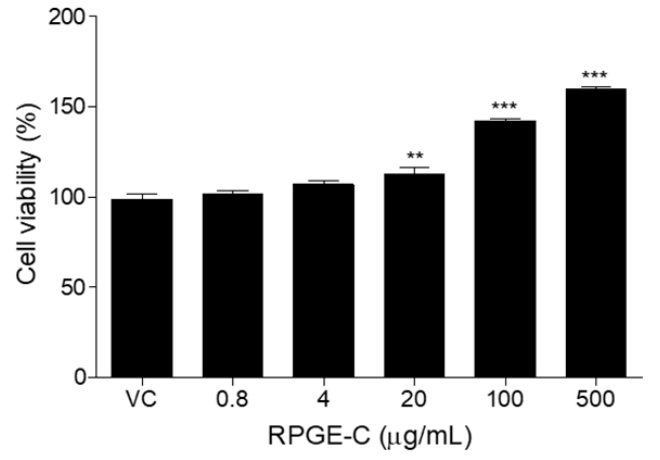

(a)

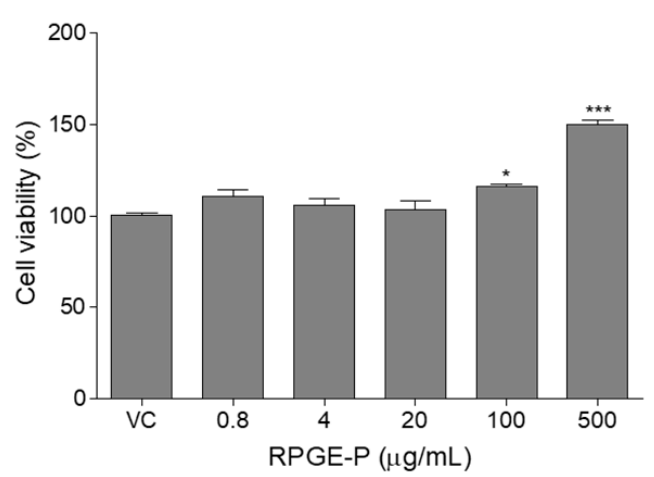

(b)

Figure 1. Effect of Red Platycodon grandiflorus Root Extract (RPGE) on cell viability in RAW 264.7 cells. (a) RPGE-concentrate (RPGE-C); (b) RPGE-powder (RPGE-P). The results are expressed as relative values to that of the vehicle-treated cells (vehicle control, VC), which is set to $100 \%$. The data are represented as the mean \pm SEM. ${ }^{*} p<0.05,{ }^{* *} p<0.01$, and ${ }^{* *} p<0.001$ when compared with the VC.

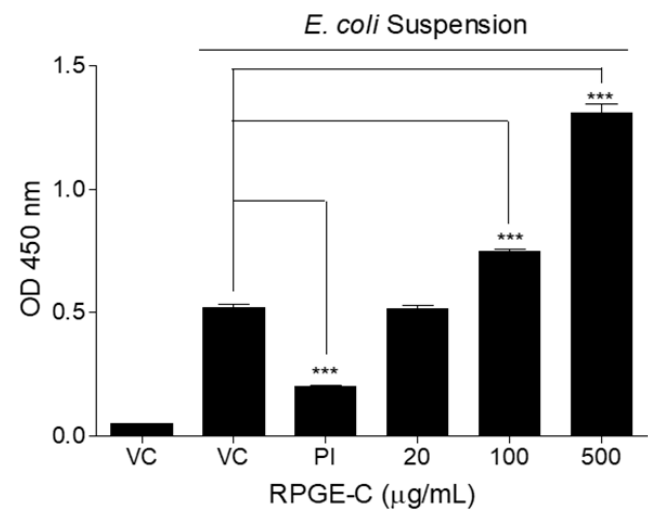

(a)

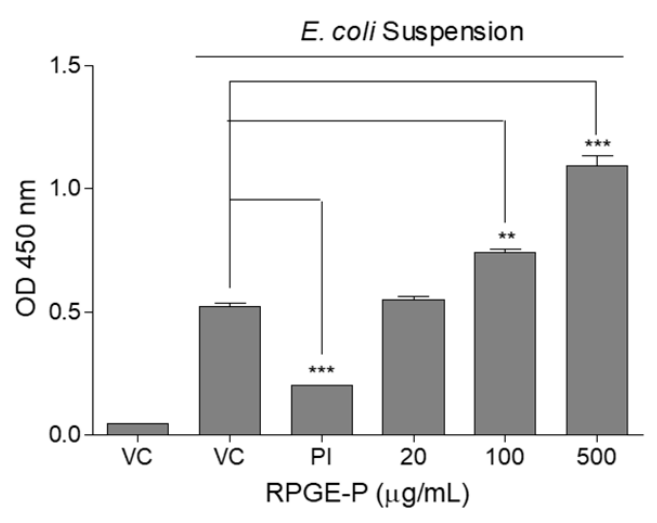

(b)

Figure 2. Effect of RPGE on the phagocytic activity of RAW 264.7 cells. (a) RPGE-C; (b) RPGE-P. PI, phagocytosis inhibitor. Values are represented as the mean \pm SEM. ${ }^{* *} p<0.01$ and ${ }^{* * *} p<0.001$ when compared with the VC treated with an E. coli suspension.

\subsection{RPGE Enhances NO Production in RAW 264.7 Cells}

The immune-enhancing effects of RPGEs were confirmed by measuring the nitrite level in cell culture medium using the Griess Reagent System. As shown in Figure 3, we observed a slight but significant enhancement in NO production after treatment with the highest concentration $(500 \mu \mathrm{g} / \mathrm{mL})$ of RPGE-C (Figure 3a) or RPGE-P (Figure 3d). Inducible nitric oxide synthase (iNOS, gene name Nos2) is a NO synthesis enzyme, and cyclooxygenase (COX-2, gene name Ptgs2) is the enzyme that converts arachidonic acid to prostaglandin E2 [16]. The Nos2 and Ptgs2 mRNA expression levels in RAW 264.7 cells were measured after RPGEs treatment. As shown in Figure 3b,c,e,f, treatment with RPGEs for $24 \mathrm{~h}$ markedly increased Nos2 and Ptgs2 mRNA expression levels compared to the vehicle control. 


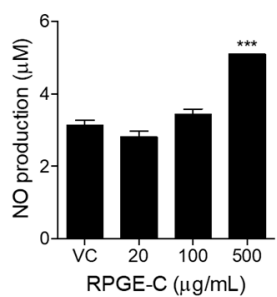

(a)

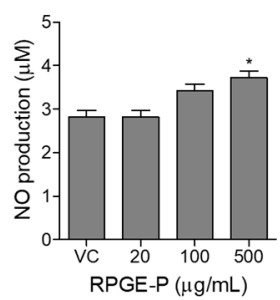

(d)

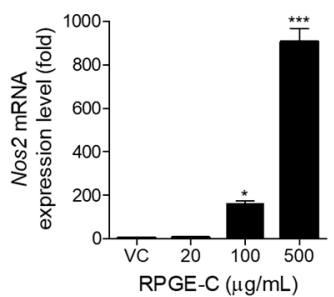

(b)

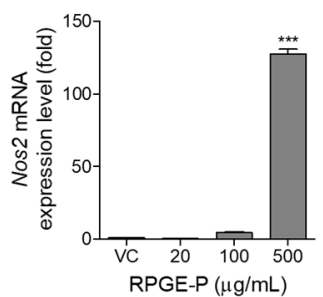

(e)

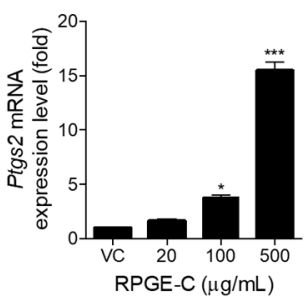

(c)

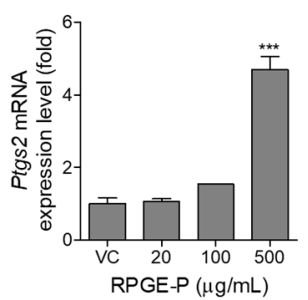

(f)

Figure 3. Effect of RPGE on NO production and related mRNA expression in RAW 264.7 cells. (a-c) RPGE-C; (d-f) RPGE-P. (a,d) NO production; (b,e) Nos2 mRNA expression level; (c,f) Ptgs2 mRNA expression level. NO, Nitric oxide. All gene expression values were normalized to $A c t b$, and the results were expressed as relative values to that of the $\mathrm{VC}$, which is set to 1 . The data are represented as the mean \pm SEM. ${ }^{*} p<0.05,{ }^{* *} p<0.01$, and ${ }^{* * *} p<0.001$ when compared with the VC.

\subsection{RPGE Increases Cytokine Levels in RAW 264.7 Cells}

To assess the modulation of cytokines related to innate immunity due to RPGE-induced macrophage activation, we measured TNF- $\alpha$, IL-1 $\beta$, and IL- 6 mRNA expression levels by real-time RT-PCR and IL-6 protein levels by ELISA. Compared to the VC, RPGE-C treatment increased the mRNA expression of TNF- $\alpha$, IL-1 $\beta$, and IL- 6 , as well as IL- 6 production, in a concentration-dependent manner (Figure 4a-d). Compared to the RPGE-C treatment, the effects of RPGE-P on the mRNA expression levels of cytokines and production level of IL-6 were less pronounced but statistically significant compared with the VC at the highest concentration $(500 \mu \mathrm{g} / \mathrm{mL}$, Figure $4 \mathrm{e}-\mathrm{h})$. These data indicated that both RPGE-C and RPGE-P play a role in cytokine regulation.

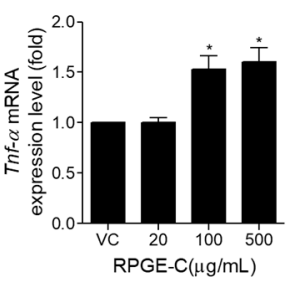

(a)

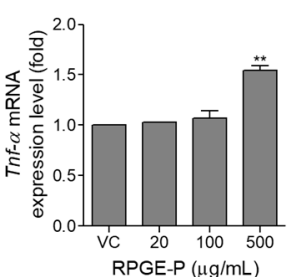

(e)

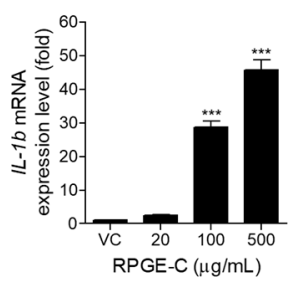

(b)

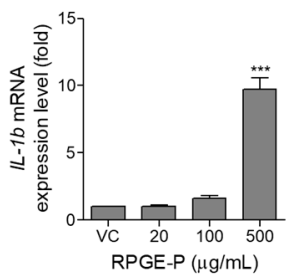

$(f)$

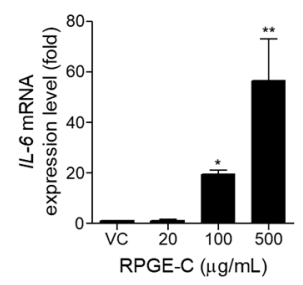

(c)

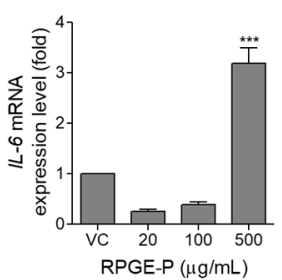

(g)

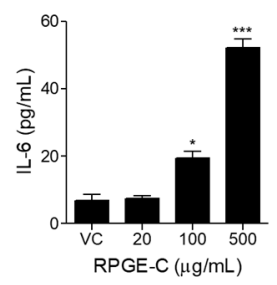

(d)

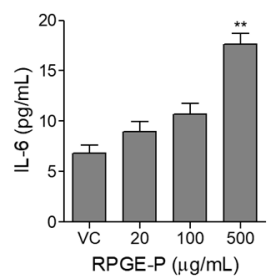

(h)

Figure 4. Effect of RPGE on cytokine levels in RAW 264.7 cells. (a-d) RPGE-C; (e-h) RPGE-P. (a,e) Tnf- $\alpha$ mRNA expression level; (b,f) $I L-1 b$ mRNA expression level; (c,g) IL-6 mRNA expression level. The gene expression values were normalized to $A c t b$, and the results were expressed as relative values to that of the VC, which is set to 1 . $(\mathbf{d}, \mathbf{h})$ IL-6 protein levels in the culture supernatant. All data are represented as the mean \pm SEM. ${ }^{*} p<0.05,{ }^{* *} p<0.01$, and ${ }^{* * *} p<0.001$ when compared with the VC. 


\subsection{RPGE Activates NF- $\kappa B$ and MAPK Signaling in RAW 264.7 Cells}

To identify the effect of RPGE on NF- $\mathrm{KB}$ signaling, we performed a transcriptional activity assay using reporter gene constructs of 3xNF-kB-binding elements in RAW 264.7 cells. Treatment with RPGE-C at concentrations of 100 and $500 \mu \mathrm{g} / \mathrm{mL}$ (Figure 5a) or RPGE-P at a concentration $500 \mu \mathrm{g} / \mathrm{mL}$ (Figure 5c) led to the induction of luciferase activity, indicating that RPGE activates NF- $\mathrm{kB}$ signaling. To understand the molecular mechanisms of the effect of RPGE on NF- $\mathrm{kB}$ signaling, we examined the phosphorylation status of NF- $\kappa B$ and I $\kappa B$ by Western blot. Our results showed that RPGE-C increased the levels of phospho-NF- $\mathrm{KB}$ and phospho-IкB $\alpha$ in a dose-dependent manner (Figure $5 b$ ). In the case of RPGE-P, phosphorylation levels of NF- $\kappa B$ and $I \kappa B \alpha$ were also increased at the highest concentration $(500 \mu \mathrm{g} / \mathrm{mL}$, Figure $5 \mathrm{~d}$ ). Moreover, the presence of an NF-kB inhibitor (pyrrolidine dithiocarbamate, PDTC) with RPGE inhibited NO production, cytokine levels, and phosphorylation of NF- $\mathrm{kB}$ (Figure S1). Therefore, our results indicated that RPGE can induce macrophage activation via NF- $\mathrm{kB}$ signaling.

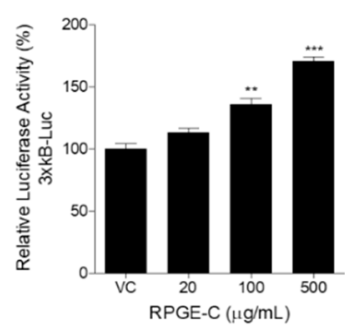

(a)

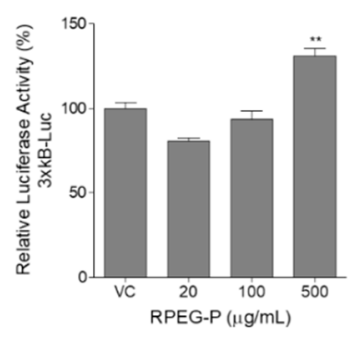

(c)

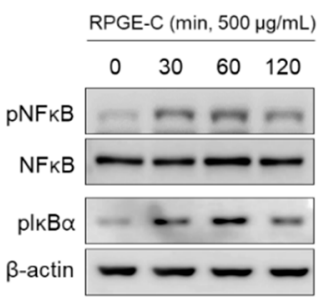

(b)

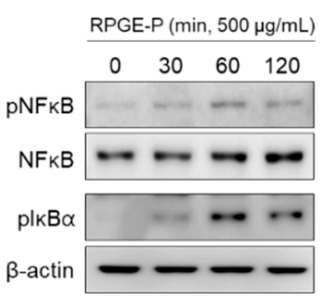

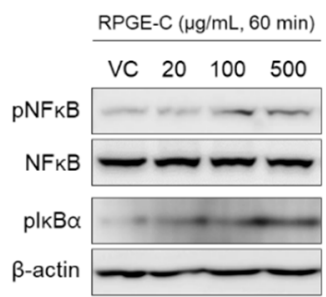

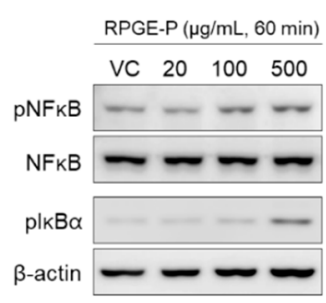

(d)

Figure 5. Effect of RPGE on NF-kB activation in RAW 264.7 cells. (a) RPGE-C; (c) RPGE-P. Luciferase activity was expressed as a relative value to that of the $\mathrm{VC}$, which is set to $100 \%$. The figures show the mean \pm SEM. ${ }^{*} p<0.05$ and ${ }^{* * *} p<0.001$. (b) The cells were treated with $500 \mu \mathrm{g} / \mathrm{mL}$ of RPGE-C for the indicated times or indicated concentrations for $60 \mathrm{~min}$ prior to Western blot analysis; (d) The cells were treated with $500 \mu \mathrm{g} / \mathrm{mL}$ of RPGE-P for the indicated times or indicated concentrations prior to Western blot analysis.

Since NF- $\mathrm{kB}$ activation is mediated via MAPK (p38 MAPK and JNK) signaling [17], we assessed whether RPGE induces MAPK activation in RAW 264.7 cells. Figure 6a,b (left) showed that the activation of p38 MAPK and JNK signaling were observed following treatment with $500 \mu \mathrm{g} / \mathrm{mL}$ of RPGE-C or RPGE-P. Maximum levels of p38 MAPK and JNK phosphorylation appeared at $30 \mathrm{~min}$ post-treatment and decreased thereafter. Not only RPGE-C but also RPGE-P treatment was shown to increase the phosphorylation level of MAPK in a concentration-dependent manner (Figure 6a,b, right). 


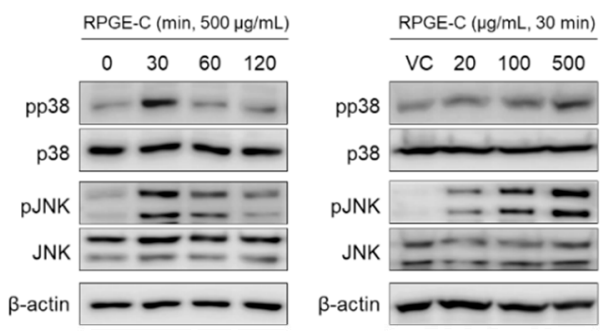

(a)
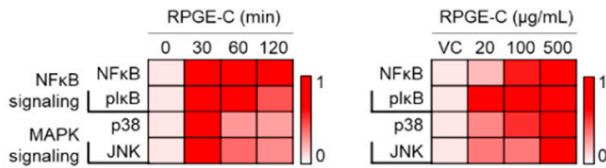

(c)
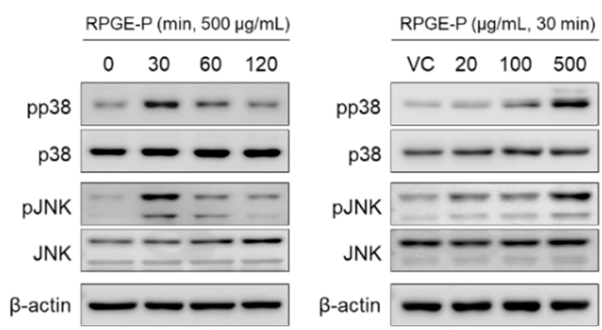

(b)
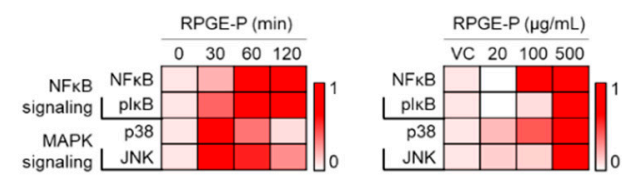

(d)

Figure 6. Effect of RPGE on MAPK signaling in RAW 264.7 cells. (a) The cells were treated with $500 \mu \mathrm{g} / \mathrm{mL}$ of RPGE-C for the indicated times or indicated concentrations for $60 \mathrm{~min}$ prior to Western blot analysis; (b) The cells were treated with $500 \mu \mathrm{g} / \mathrm{mL}$ of RPGE-P for the indicated times or indicated concentrations for $60 \mathrm{~min}$ prior to Western blot analysis; (c) The time-response and concentration-response relationships of RPGE-C were represented as heat maps. (d) The time-response and concentration-response relationships of RPGE-P were represented as heat maps.

\subsection{RPGE-Induced NF- $\kappa B$ Activation Is Associated with p38 MAPK in RAW 264.7 Cells}

To identify the association between the MAPK and NF- $\mathrm{KB}$ signaling by RPGE, we performed a luciferase assay using SB203580 (a p38 MAPK inhibitor) and SP600125 (a JNK inhibitor). Firstly, we confirmed that these inhibitors did not influence luciferase activity (Figure 7a). Treatment with $10 \mu \mathrm{M}$ SB203580 (Figure 7b) markedly reduced RPGE-C or RPGE-P induced NF- $\mathrm{KB}$ signaling. however, cotreatment with $10 \mu \mathrm{M} \mathrm{SP600125} \mathrm{(Figure} \mathrm{7c)} \mathrm{had} \mathrm{no} \mathrm{effect} \mathrm{on} \mathrm{NF- \kappa B} \mathrm{signaling.} \mathrm{These} \mathrm{results} \mathrm{suggested}$ that the $\mathrm{p} 38 \mathrm{MAPK}$ is associated with RPGE-induced NF- $\mathrm{kB}$ activation.

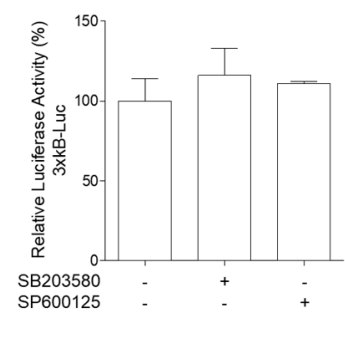

(a)

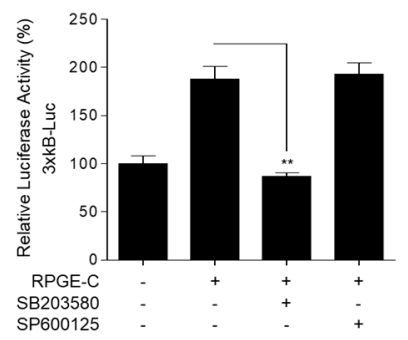

(b)

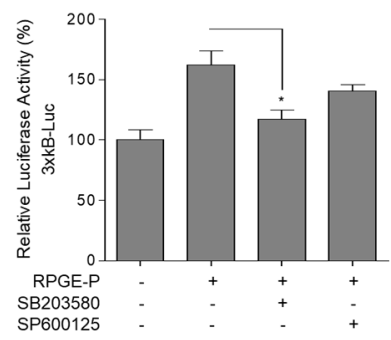

(c)

Figure 7. Effect of MAPK signaling on NF- $\mathrm{kB}$ activation in RAW 264.7 cells. (a) without RPGEs; (b) RPGE-C; (c) RPGE-P. Luciferase activity was expressed as a relative value to that of the VC (-), which is set to $100 \%$. The figures show the mean \pm SEM. ${ }^{*} p<0.05$ and ${ }^{* *} p<0.01$ when compared with cells treated with RPGEs alone. SB203580, p38 MAPK inhibitor and SP600125, JNK inhibitor.

\subsection{RPGE Induces Cell Proliferation and Increases IL-10 Expression Levels in Mouse Splenocytes}

To assess the immune-enhancing effect of RPGE in splenocytes, we isolated whole spleen cells from mice. The cell viability of splenocytes treated with reference mitogens (T-lymphocyte mitogen, Con A or B-lymphocyte mitogen, LPS) was increased by approximately $230 \%$ and $300 \%$ of the VC $(100 \%)$, respectively. Viable cell populations were gradually increased by treatment with RPGE-C in a concentration-dependent manner (Figure 8a) and by treatment with the highest concentration (500 $\mu \mathrm{g} / \mathrm{mL}$ ) of RPGE-P (Figure 8c). To determine whether RPGE could affect IL-10 production, we investigated the secretion of IL-10 in isolated mouse splenocytes. Both RPGE-C and RPGE-P 
treatment induced the secretion of IL-10 (Figure 8b,d), which suggested that RPGE could enhance immunity via modulation of immune lymphocytes, such as B cells.

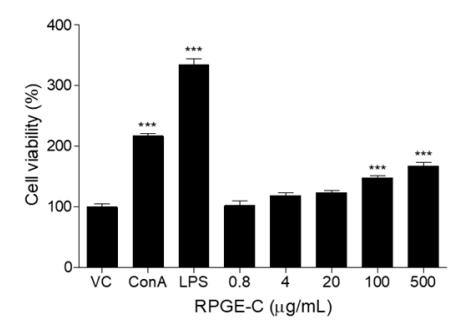

(a)

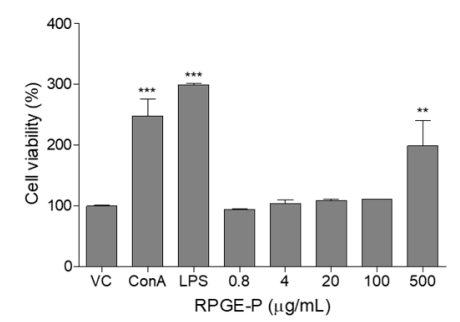

(c)

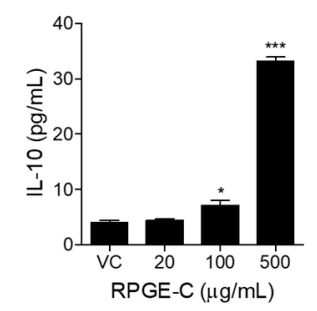

(b)

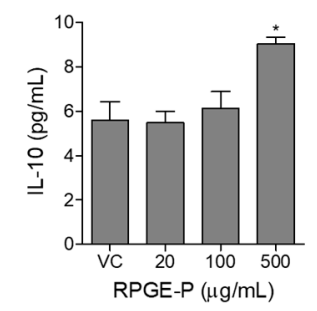

(d)

Figure 8. Effect of RPGE on cell viability and secretion of IL-10 in mouse splenocytes. (a) Mouse splenocytes were incubated with Con A $(5 \mu \mathrm{g} / \mathrm{mL})$, LPS $(1 \mu \mathrm{g} / \mathrm{mL})$, or various concentrations of RPGE-C; (c) Mouse splenocytes were incubated with Con A $(5 \mu \mathrm{g} / \mathrm{mL})$, LPS $(1 \mu \mathrm{g} / \mathrm{mL})$, or various concentrations of RPGE-P. Con A, Concanavalin A; LPS, lipopolysaccharide. The results were expressed as a relative value to that of $\mathrm{VC}$, which is set to $100 \%$. The data are represented as the mean \pm SEM. ${ }^{* *} p<0.01$ and ${ }^{* * *} p<0.001$ as when compared with the VC. (b) IL-10 protein level (RPGE-C); (d) IL-10 protein level (RPGE-P). All data are represented as the mean \pm SEM. ${ }^{*} p<0.05$ and ${ }^{* * *} p<0.001$ as when compared with the VC.

\section{Discussion}

The host immune system plays a crucial role in defending or inhibiting several pathological conditions, such as infections and tumors $[18,19]$. however, the most important aspect of the immunity is the balance between activation and inhibition. There are some plant extracts or their components that have been reported to have an immunostimulant effects under normal conditions, whereas they have an immunosuppressing effect in inflammatory environments, such as LPS treatment [7,20-22].

In the present study, we have revealed the immune-enhancing effect of RPGE. RPGE-C or RPGE-P treatment significantly increased phagocytic activity in macrophages, as well as NO production and mRNA expression levels of innate immune-related cytokines. Moreover, our previous studies have also confirmed the mitigating effects of RPGE on the LPS-induced inflammatory response in splenocytes isolated from mice [8]. Therefore, we concluded that RPGE has immunomodulatory properties in immune cells.

NF- $K B$ and MAPK signaling play a crucial role in immune responses $[1,4,23,24]$. however, the relationship between the two signaling pathways remains unclear. here, our results show that RPGE stimulates the immune responses in RAW 264.7 cells via two different mechanisms. RPGE has shown to increase phosphorylation levels of NF- $\mathrm{KB}$, as well as increase p38 MAPK-induced NF- $\mathrm{kB}$ activation. The inhibition of NF- $\mathrm{kB}$ and $\mathrm{p} 38$ reduced RPGE-induced macrophage activation (Figure S1). Therefore, our results suggest that RPGE is a promising functional food for enhancing immunity. Moreover, further studies on the immunomodulatory effect of RPGE may further understanding of the molecular mechanisms underlying the immune response.

The functional food industry has used various processing methods to increase the active ingredients of raw materials [25-27]. Approximately 30 different active ingredients of PG are known [28], including 
platycodin D, the representative saponin in PG (Figure S2A). In this study, we steamed and dried raw PG several times, thereby increasing the content of platycodin $D$ by approximately three times when compared to white PG (Figure S2B). This process is similar to the increase in the saponin content of red ginseng compared to white ginseng $[5,26,27]$. Moreover, this increase in the content of active ingredients may further improve the functionality of PG.

To determine the cellular mechanism of the immune-enhancing effect of RPGE, we used the murine macrophage cell line, RAW 264.7. The viable cell population was increased by RPGE treatment (Figure 1a,b). The mean proliferation rates after treatment with the highest concentration of RPGE-C and RPGE-P $(500 \mu \mathrm{g} / \mathrm{mL})$ were increased by approximately $60 \%$ and $50 \%$ compared to the VC, respectively. Thus, our results demonstrate that RPGEs induce macrophage cell proliferation in RAW 264.7 cells. Interestingly, extracellular-signal-regulated kinase (ERK) activity was increased in cells treated with RPGE-C (Figure S3A) or RPGE-P (Figure S3B). ERK is a family of MAPK that plays a role in regulating cell growth signals $[29,30]$. When cells were cotreated with RPGEs and an ERK inhibitor (PD98059), NF-KB activity was increased (Figure S3C). This is consistent with the results obtained in the previous study, which showed that ERK inhibition activates NF- $\kappa B$ [31]. These results suggest that RPGE-induced ERK activation in RAW 264.7 cells might regulate cell proliferation rather than immune activity.

When comparing RPGE-C and RPGE-P, both extracts showed dose-dependent-induced phagocytosis. however, for most of pharmaceutical activities, including cytokines and NF- KB, RPGE-C was more effective at lower concentrations than RPGE-P. These results are typical, as the RPGE-P was prepared by mixing with dextrin, and experiments with higher doses of RPGE-P are not possible due to the limited solubility. Moreover, these effects were not ascribed to processing but due to variations in their content, therefore it is suggested that more powder should be consumed than concentrate for the same effect. In conclusion, we showed the immunostimulatory effects of RPGE and its mechanisms, both in vitro and ex vivo. however, further studies are necessary to confirm the effects of RPGEs in animal models and humans.

Supplementary Materials: The following are available online at http://www.mdpi.com/2076-3417/10/16/5457/s1, Figure S1: Effects of PDTC or SB203580 on RPGE-induced immunomodulation in RAW 264.7 cells.; Figure S2: high-performance liquid chromatography (HPLC) analysis of RPGE.; Figure S3: Effect of RPGE on ERK activation in RAW 264.7 cells.

Author Contributions: E.-J.P. and h.-J.L. designed the study. E.-J.P., Y.-S.L. and S.-H.L. contributed methodology. E.-J.P., Y.-S.L., S.M.K., J.-H.Y. and A.J.J. performed the experiments. h.C.J. performed the experiment of Figure S2. E.-J.P. analyzed the data. E.-J.P. wrote the manuscript. h.-J.L. supervised the project and revised the manuscript. All authors have read and agreed to the published version of the manuscript.

Funding: This work was supported by the "Cooperative Research Program for Agriculture Science and Technology Development (Project No. PJ01381002)" Rural Development Administration, Republic of Korea.

Acknowledgments: We are grateful to Ju-hong Jeon (Seoul National University) for providing the $3 \times \kappa \mathrm{B}-\mathrm{Luc}$ reporter gene plasmids.

Conflicts of Interest: The authors declare no conflict of interest.

\section{References}

1. Kim, Y.S.; Kim, E.K.; Nawarathna, W.; Dong, X.; Shin, W.B.; Park, J.S.; Moon, S.H.; Park, P.J. Immune-Stimulatory Effects of Althaea rosea Flower Extracts through the MAPK Signaling Pathway in RAW264.7 Cells. Molecules 2017, 22, 679. [CrossRef]

2. Kim, G.T.; Tran, N.K.; Choi, E.H.; Song, Y.J.; Song, J.H.; Shim, S.M.; Park, T.S. Immunomodulatory Efficacy of Standardized Annona muricata (Graviola) Leaf Extract via Activation of Mitogen-Activated Protein Kinase Pathways in RAW 264.7 Macrophages. Evi. Based Complementary Altern. Med. 2016, 2016, 2905127.

3. Chun, S.H.; Lee, H.A.; Lee, K.B.; Kim, S.H.; Park, K.Y.; Lee, K.W. Effects of Glycated Whey Protein Concentrate on Pro-inflammatory Cytokine Expression and Phagocytic Activity in RAW264.7 Macrophages. Biol. Pharm. Bull. 2016, 39, 199-206. [CrossRef] 
4. Hayden, M.S.; West, A.P.; Ghosh, S. NF-kappaB and the immune response. Oncogene 2006, 25, 6758-6780. [CrossRef]

5. Shin, M.S.; Song, J.H.; Choi, P.; Lee, J.H.; Kim, S.Y.; Shin, K.S.; ham, J.; Kang, K.S. Stimulation of Innate Immune Function by Panax ginseng after heat Processing. J. Agric. Food. Chem. 2018, 66, 4652-4659. [CrossRef]

6. Ma, D.; Zhang, R.N.; Wen, Y.; Yin, W.N.; Bai, D.; Zheng, G.Y.; Li, J.S.; Zheng, B.; Wen, J.K. 1,25(OH)2D3-induced interaction of vitamin D receptor with p50 subunit of NF-kappaB suppresses the interaction between KLF5 and p50, contributing to inhibition of LPS-induced macrophage proliferation. Biochem. Biophys. Res. Commun. 2017, 482, 366-374. [CrossRef]

7. Ji, K.Y.; Kim, K.M.; Kim, Y.H.; Im, A.R.; Lee, J.Y.; Park, B.; Na, M.; Chae, S. The enhancing immune response and anti-inflammatory effects of Anemarrhena asphodeloides extract in RAW 264.7 cells. Phytomed. Int. J. Phytother. Phytopharmacol. 2019, 59, 152789.

8. Park, E.J.; Lee, Y.S.; Jeong, H.C.; Lee, S.H.; Lee, H.J. Mitigation effects of red Platycodon grandiflorum extract on lipopolysaccharide-induced inflammation in splenocytes isolated from mice. J. Nutr. health 2019, 52, 6. [CrossRef]

9. Mebius, R.E.; Kraal, G. Structure and function of the spleen. Nat. Rev. Immunol. 2005, 5, 606-616. [CrossRef]

10. Park, M.; Park, S.Y.; Lee, H.J.; Kim, C.E. A Systems-Level Analysis of Mechanisms of Platycodon grandiflorum Based on A Network Pharmacological Approach. Molecules 2018, 23, 2841. [CrossRef]

11. Li, W.; Tian, Y.H.; Liu, Y.; Wang, Z.; Tang, S.; Zhang, J.; Wang, Y.P. Platycodin D exerts anti-tumor efficacy in h22 tumor-bearing mice via improving immune function and inducing apoptosis. J. Toxicol. Sci. 2016, 41, 417-428. [CrossRef]

12. Xie, Y.; Ye, Y.P.; Sun, H.X.; Li, D. Contribution of the glycidic moieties to the haemolytic and adjuvant activity of platycodigenin-type saponins from the root of Platycodon grandiflorum. Vaccine 2008, 26, 3452-3460. [CrossRef]

13. Park, M.; Yoo, J.H.; Lee, Y.S.; Park, E.J.; Lee, H.J. Ameliorative effects of black ginseng on nonalcoholic fatty liver disease in free fatty acid-induced hepG2 cells and high-fat/high-fructose diet-fed mice. J. Ginseng Res. 2020, 44, 350-361. [CrossRef]

14. Ghosh, S.; howe, N.; Volk, K.; Tati, S.; Nickerson, K.W.; Petro, T.M. Candida albicans cell wall components and farnesol stimulate the expression of both inflammatory and regulatory cytokines in the murine RAW264.7 macrophage cell line. FEMS Immunol. Med. Microbiol. 2010, 60, 63-73. [CrossRef]

15. Chun, J.N.; Park, S.; Lee, S.; Kim, J.K.; Park, E.J.; Kang, M.; Kim, H.K.; Park, J.K.; So, I.; Jeon, J.H. Schisandrol $\mathrm{B}$ and schisandrin B inhibit TGFbeta1-mediated NF-kappaB activation via a Smad-independent mechanism. Oncotarget 2018, 9, 3121-3130. [CrossRef]

16. Murakami, A.; Ohigashi, H. Targeting NOX, INOS and COX-2 in inflammatory cells: chemoprevention using food phytochemicals. Int. J. Cancer 2007, 121, 2357-2363. [CrossRef]

17. Lee, J.; Choi, J.W.; Sohng, J.K.; Pandey, R.P.; Park, Y.I. The immunostimulating activity of quercetin 3-O-xyloside in murine macrophages via activation of the ASK1/MAPK/NF-kappaB signaling pathway. Int. Immunopharmacol. 2016, 31, 88-97. [CrossRef] [PubMed]

18. Chaplin, D.D. Overview of the immune response. J. Allergy Clin. Immunol. 2010, 125, S3-S23. [CrossRef]

19. Pandya, P.H.; Murray, M.E.; Pollok, K.E.; Renbarger, J.L. The Immune System in Cancer Pathogenesis: Potential Therapeutic Approaches. J. Immunol. Res. 2016, 2016, 4273943. [CrossRef]

20. Ghonime, M.; Emara, M.; Shawky, R.; Soliman, H.; El-Domany, R.; Abdelaziz, A. Immunomodulation of RAW 264.7 murine macrophage functions and antioxidant activities of 11 plant extracts. Immunol. Invest. 2015, 44, 237-252. [CrossRef]

21. Choi, E.Y.; Lee, S.S.; hyeon, J.Y.; Choe, S.H.; Keum, B.R.; Lim, J.M.; Park, D.C.; Choi, I.S.; Cho, K.K. Effects of beta-Glucan on the Release of Nitric Oxide by Macrophages Stimulated with Lipopolysaccharide. Asian-Australas. J. Anim. Sci. 2016, 29, 1664-1674. [CrossRef]

22. Checker, R.; Patwardhan, R.S.; Sharma, D.; Menon, J.; Thoh, M.; Bhilwade, H.N.; Konishi, T.; Sandur, S.K. Schisandrin B exhibits anti-inflammatory activity through modulation of the redox-sensitive transcription factors Nrf2 and NF-kappaB. Free Radical Biol. Med. 2012, 53, 1421-1430. [CrossRef]

23. Gerondakis, S.; Siebenlist, U. Roles of the NF-kappaB pathway in lymphocyte development and function. Cold Spring harbor Perspect. Biol. 2010, 2, a000182. [CrossRef] [PubMed] 
24. Dong, C.; Davis, R.J.; Flavell, R.A. MAP kinases in the immune response. Annu. Rev. Immunol. 2002, 20, 55-72. [CrossRef]

25. Chao, J.; Dai, Y.; Cheng, H.Y.; Lam, W.; Cheng, Y.C.; Li, K.; Peng, W.H.; Pao, L.H.; hsieh, M.T.; Qin, X.M.; et al. Improving the Concentrations of the Active Components in the herbal Tea Ingredient, Uraria crinita: The Effect of Post-harvest Oven-drying Processing. Sci. Rep. 2017, 7, 38763. [CrossRef]

26. Shin, J.H.; Park, Y.J.; Kim, W.; Kim, D.O.; Kim, B.Y.; Lee, H.; Baik, M.Y. Change of Ginsenoside Profiles in Processed Ginseng by Drying, Steaming, and Puffing. J. Microbiol. Biotechnol. 2019, 29, 222-229. [CrossRef]

27. Lee, S.M.; Bae, B.S.; Park, H.W.; Ahn, N.G.; Cho, B.G.; Cho, Y.L.; Kwak, Y.S. Characterization of Korean Red Ginseng (Panax ginseng Meyer): history, preparation method, and chemical composition. J. Ginseng Res. 2015, 39, 384-391. [CrossRef]

28. Choi, Y.H.; Yoo, D.S.; Choi, C.W.; Cha, M.R.; Kim, Y.S.; Lee, H.S.; Lee, K.R.; Ryu, S.Y. Platyconic acid A, a genuine triterpenoid saponin from the roots of Platycodon grandiflorum. Molecules 2008, 13, 2871-2879. [CrossRef]

29. Zhang, W.; Liu, H.T. MAPK signal pathways in the regulation of cell proliferation in mammalian cells. Cell Res. 2002, 12, 9-18. [CrossRef]

30. Richardson, E.T.; Shukla, S.; Nagy, N.; Boom, W.H.; Beck, R.C.; Zhou, L.; Landreth, G.E.; harding, C.V. ERK Signaling Is Essential for Macrophage Development. PLoS ONE 2015, 10, e0140064. [CrossRef]

31. Funakoshi, M.; Tago, K.; Sonoda, Y.; Tominaga, S.; Kasahara, T. A MEK inhibitor, PD98059 enhances IL-1-induced NF-kappaB activation by the enhanced and sustained degradation of IkappaBalpha. Biochem. Biophys. Res. Commun. 2001, 283, 248-254. [CrossRef]

(C) 2020 by the authors. Licensee MDPI, Basel, Switzerland. This article is an open access article distributed under the terms and conditions of the Creative Commons Attribution (CC BY) license (http://creativecommons.org/licenses/by/4.0/). 